\title{
Metal-like conductivity in microbial nanowires
}

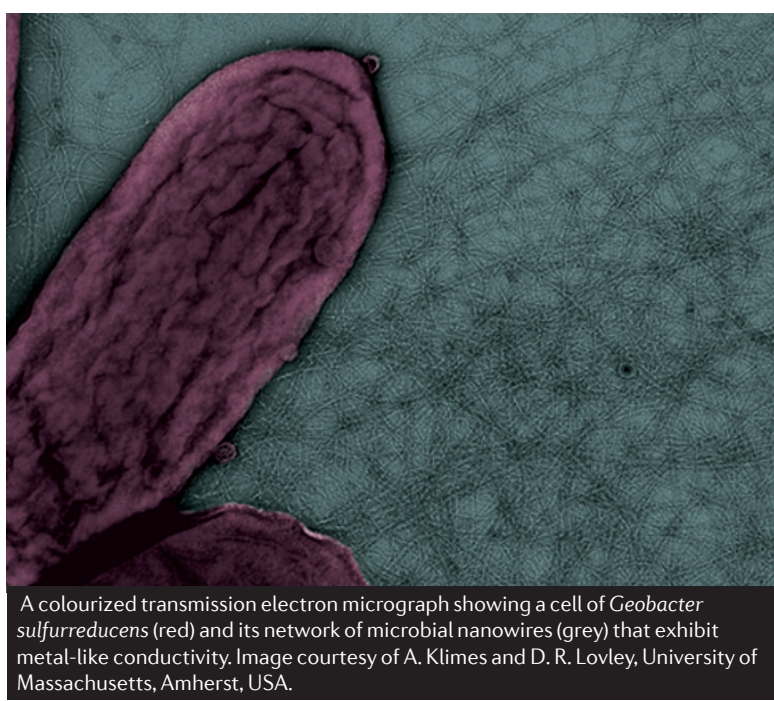

The bacterium Geobacter sulfurreducens can obtain energy for growth by oxidizing organic compounds and transferring the resulting electrons through long filaments, known as pili or microbial nanowires, to the surface of iron oxide particles. However, it was unclear how the electron flow takes place, as the pili are composed of oligomeric pilin proteins, a material that was expected to be an electrical insulator. Writing in Nature Nanotechnology, Malvankar et al. now show that microbial nanowires behave as metal-like conductors and allow electron transport through pilins across long distances.
Previous studies of microbial nanowires gave contrasting results concerning the possible role of pilins and outer-surface $c$-type cytochromes in electron transfer. To investigate the conductivity of these nanowires, Malvankar et al. grew biofilms of $G$. sulfurreducens in a microbial fuel cell in which the anode (which serves as the electron acceptor to support growth) was composed of two gold electrodes separated by a non-conductive gap of $50 \mu \mathrm{m}$. The production of current and the conductivity between anode and cathode increased over time as cells grew in a biofilm that spread across the gap. Biofilms were conductive over distances of more than $1 \mathrm{~cm}$, with conductivity values $\left(5 \mathrm{mS} \mathrm{cm}^{-1}\right)$ that were comparable to those of synthetic organic metals such as polyaniline and polyacetylene. By manipulating the medium composition and using various $G$. sulfurreducens strains (including one that lacked four abundant outer-surface $c$-type cytochromes), the authors found that biofilm conductivity correlated with pilin content. Furthermore, purified pili showed a conductivity that was comparable to that of peeled biofilms, even after treatment with the cytochrome-denaturing agent $\beta$-mercaptoethanol. These results indicate that G. sulfurreducens pili can account for the electron flow through biofilms.

The conductivities of biofilms and pili displayed several properties that are typical of organic metals; for example, they changed with temperature in a characteristic way. These results, together with X-ray diffraction patterns of purified pili, led the authors to suggest that the conductive properties of microbial nanowires can be explained by $\pi$-orbital overlap and charge delocalization between stacked aromatic rings of amino acids within and between pilin subunits.

Although more studies are needed for a better understanding of the conduction mechanism, the finding that bacterial protein filaments can conduct electricity in a way that is comparable to that found in synthetic polymers may lead to cheaper, non-toxic electronic materials that could be combined with other biomaterials and used in moist environments.

\section{Cesar Sanchez}

\section{ORIGINAL RESEARCH PAPER}

Malvankar, N. S. et al. Tunable metallic-like conductivity in microbial nanowire networks. Nature Nanotech. 7 Aug 2011 (doi:10.1038/nnano.2011.119) FURTHER READING Logan, B. E. Exoelectrogenic bacteria that power microbial fuel cells. Nature Rev. Microbiol. 7, 375-381 (2009) 